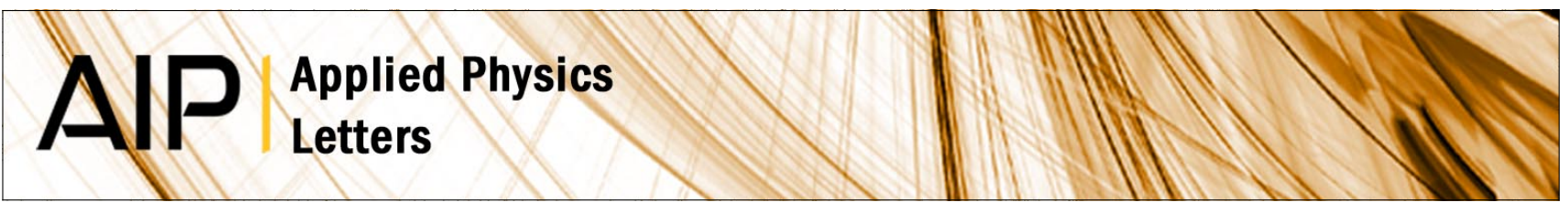

\title{
Multifunctional silicon inspired by a wing of male Papilio ulysse
}

Sang H. Yun, Hyung-Seok Lee, Young Ha Kwon, Mats Göthelid, Sang Mo Koo et al.

Citation: Appl. Phys. Lett. 100, 033109 (2012); doi: 10.1063/1.3664636

View online: http://dx.doi.org/10.1063/1.3664636

View Table of Contents: http://apl.aip.org/resource/1/APPLAB/v100/i3

Published by the American Institute of Physics.

\section{Related Articles}

Nonlinear behavior of photoluminescence from silicon particles under two-photon excitation Appl. Phys. Lett. 99, 251105 (2011)

About the internal pressure in cavities derived from implantation-induced blistering in semi-conductors J. Appl. Phys. 110, 114903 (2011)

Structural evolution of implanted vicinal $\mathrm{Si}(111)$ during annealing via analysis of the dipole contribution J. Appl. Phys. 110, 103520 (2011)

Positive or negative gain: Role of thermal capture cross sections in impurity photovoltaic effect J. Appl. Phys. 110, 104508 (2011)

Photoemission induced bias in two-dimensional silicon pn junctions

Appl. Phys. Lett. 99, 202107 (2011)

\section{Additional information on Appl. Phys. Lett.}

Journal Homepage: http://apl.aip.org/

Journal Information: http://apl.aip.org/about/about_the_journal

Top downloads: http://apl.aip.org/features/most_downloaded

Information for Authors: http://apl.aip.org/authors

\section{ADVERTISEMENT}

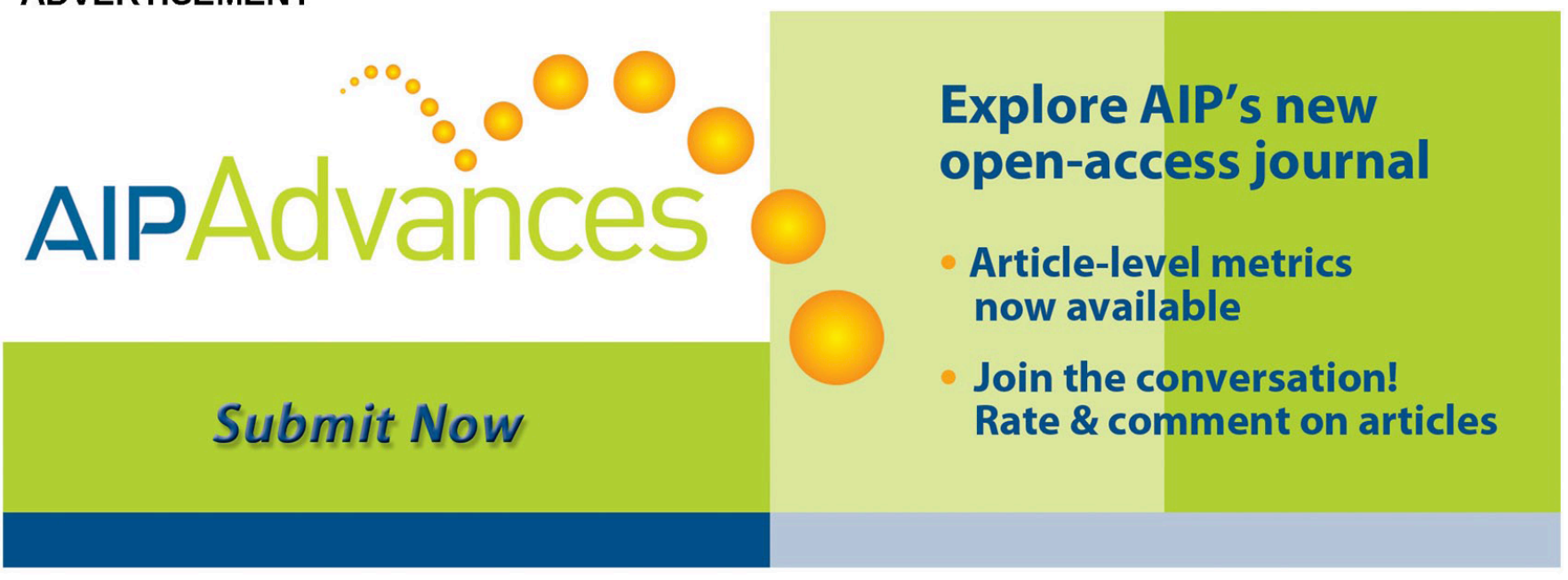




\title{
Multifunctional silicon inspired by a wing of male Papilio ulysse
}

\author{
Sang H. Yun, ${ }^{1, a)}$ Hyung-Seok Lee, ${ }^{2}$ Young Ha Kwon, ${ }^{3}$ Mats Göthelid, ${ }^{1}$ Sang Mo Koo, ${ }^{4}$ \\ Lars Wågberg, ${ }^{5}$ Ulf $O$. Karlsson, ${ }^{1}$ and Jan Linnros ${ }^{1}$ \\ ${ }_{1}^{1}$ Materials Physics, Royal Institute of Technology, Kista 16440, Sweden \\ ${ }^{2}$ Microsystems Technology Laboratories, Massachusetts Institute of Technology, Cambridge, \\ Massachusetts 02139, USA \\ ${ }^{3}$ Department of Mechanical Engineering, Kyung Hee University, Suwon 446-701, South Korea \\ ${ }^{4}$ Department of Electronic Materials, Kwangwoon University, Seoul 139-701, South Korea \\ ${ }^{5}$ Fibre and Polymer Technology, Royal Institute of Technology, Stockholm 10044, Sweden
}

(Received 14 September 2011; accepted 2 November 2011; published online 17 January 2012)

\begin{abstract}
Effective entrapment of air and light is a key element for maintaining stable superhydrophobicity and enhancing anti-reflection or absorption. Inspired by a wing of male Papilio ulysse having a unique structure for enabling effective trapping of air and light, we demonstrate that the structure consisting of well-defined multilayer decorated by nanostructures can be obtained on a silicon wafer by a simple microelectromechanical process, consequently resulted in stable superhydrophobocity under static and dynamic conditions, and strong wideband optical absorption. (ㄷ 2012 American Institute of Physics. [doi:10.1063/1.3664636]
\end{abstract}

Hierarchical nano- and microstructure is a common feature in biological surfaces, exhibiting remarkable properties such as superhydrophobic, self-cleaning, anti-reflective, and strong absorbing characteristics as observed in a wing of male Papilio ulysse. ${ }^{1-3}$ It is known that the observed properties are mainly induced by air or light entrapment in the structures. As such, according to Cassie's model describing the wetting of inhomogeneous surfaces, a rough solid surface can maintain a composite interface (liquid-air-solid) formed by trapped air between a water drop and a solid, ${ }^{4}$ inducing hydrophobicity by partial sitting of a water drop on air. However, the composite interface is usually quite unstable towards external stimuli, consequently leading to loss of hydrophobocity ${ }^{5-9}$ Thus, stabilizing the composite interface is crucial to maintain long-term stability of roughness induced hydrophobicity, which is closely related to the ability of air-trapping in a rough surface. In addition, to enhance anti-reflectance and absorption in a broad range of the sun spectrum light should be effectively trapped by surface features. In this regard, effective trapping of air or light is a key component for mimicking such biological surfaces.

Although a multitrap structure can be considered as a potential route to effective trapping of air or light, the fabrication difficulties hinder the realization of the structures. The structures particularly generated on inorganic materials are highly desirable for practical applications due to their chemical and mechanical stability in harsh environments. Moreover, lacking well-defined model structures limit to in-depth investigations of the observed properties.

A wing of male Papilio ulysse provide an important insight into the generation of effective trapping air and light because it consists of a complex multilayer covered by nanostructures. Inspried by the unique structure of the wing, here we report a simple technique for fabricating a multiple trap structure (MTS) on silicon as a highly applicable material for various functional surfaces and devices.

${ }^{\text {a)} E l e c t r o n i c ~ m a i l: ~ s h y @ k t h . s e . ~}$
To fabricate the MTS, we used a deep reactive ion etching (DRIE) technique with the Bosch process ${ }^{10}$ which is commonly utilized for the fabrication of deep pore or trench arrays. In the etching process, however, severe undercut beneath an etching mask often causes a collapse of the structure formed. Furthermore, a sequence of alternating passivation and etch cycles in the Bosch process produces easily the so-called "scalloping" on the sidewall of pores or trenches, which should be avoided for many applications. ${ }^{11,12}$ In the present experiment, our approach for fabricating the MTS was to intentionally use the detrimental effects, i.e., undercut and scalloping. A boron-doped $\mathrm{Si}$ (100) wafer was cleaned in the $\mathrm{H}_{2} \mathrm{SO}_{4}: \mathrm{H}_{2} \mathrm{O}_{2}=3: 1$ solution. A $1 \mu \mathrm{m}$-thick silicon oxide layer as an etching mask was thermally deposited on the wafer. Patterning a feature of $0.7 \mu \mathrm{m}$ in pore size and $1.4 \mu \mathrm{m}$ in pitch size on the $\mathrm{SiO}_{2}$ was performed by standard lithography, followed by DRIE. $\mathrm{SF}_{6}$ and $\mathrm{C}_{4} \mathrm{~F}_{8}$ were used as an etching and passivation gas, respectively. The etching and passivation cycles were switched in a range of 6-9 $\mathrm{s}$. Total processing time was $10 \mathrm{~min}$. The flow rates of $\mathrm{SF}_{6}$ and $\mathrm{C}_{4} \mathrm{~F}_{8}$, and platen power were precisely tuned by ramping, while coil power was fixed at $600 \mathrm{~W}$. A flow rate of $\mathrm{C}_{4} \mathrm{~F}_{8}$ was set at $80 \mathrm{sccm}$ (standard cubic centimeters per minute) and gradually increased at a rate of $1.5 \mathrm{sccm}$. A flow rate of $\mathrm{SF}_{6}$ was ramped down from $130 \mathrm{sccm}$ at a rate of $0.5 \mathrm{sccm}$. To improve sidewall profile, platen power was increased from $12 \mathrm{~W}$ to $17 \mathrm{~W}$ at a rate of $0.5 \mathrm{~W} / \mathrm{min}$ during the process. In general, coating low-surface-energy organic material is required for achieving artificial superhydrophobic surfaces. However, the organic materials are not robust in harsh environments. Thus, the artificial superhydrophobic surfaces achieved only by texturing are highly desirable. In this respect, low-surface-energy material was not coated on the pristine MTS. To rule out wetting properties affected by the residual polymeric $\mathrm{C}_{4} \mathrm{~F}_{8}$ on the pristine structure, we used hydrofluoric acid and potassium hydroxide ( $\mathrm{KOH}$ ) solution for removing $\mathrm{C}_{4} \mathrm{~F}_{8}$.

Fig. 1 shows representative scanning electron microscope (SEM) images and a schematic illustration of the MTS. The MTS consists of multilayer decorated by 

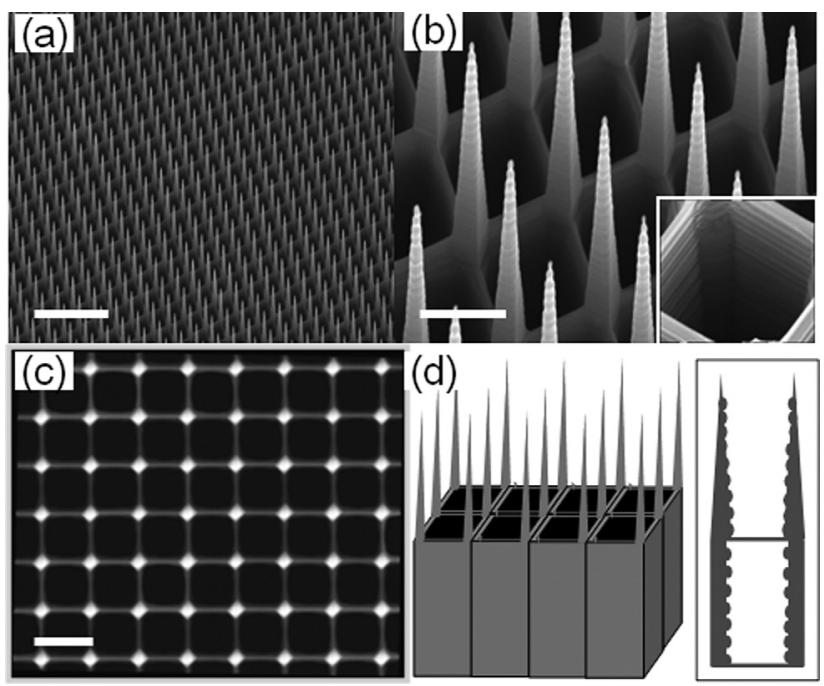

FIG. 1. SEM images of the MTS. (a) Large area, scale bar $(5 \mu \mathrm{m})$ and (b) magnified view showing the formation of nanobumps and nanogrooves on the sidewall of nanocones and micropore structures, scale bar $(1 \mu \mathrm{m})$. (c) Top view, scale bar $(1 \mu \mathrm{m})$. (d) Schematic illustrations of the MTS.

nanostructures. The rectangular nanocones are placed on the top edges of the micropore structure, which were resulted from selective etching by controlling undercut at the initial etching stage. The sidewalls of the nanocones and micropores are covered by a combination of nanobumps and nanogrooves which was generated from the scalloping. The base length and height of the nanocone are $200 \mathrm{~nm}$ and $2 \mu \mathrm{m}$, respectively. The depth of the micropore is $1 \mu \mathrm{m}$ and the spacing of the nanobumps is about $25 \mathrm{~nm}$.

$\mathrm{X}$-ray photoelectron spectroscopy (XPS) using $\mathrm{Mg} \mathrm{K} \alpha$ radiation $(\mathrm{h} v=1254 \mathrm{eV}$ ) was performed to measure the elemental composition of the MTS surface (Fig. 2). The MTS contained negligible amounts of $\mathrm{C}$. This was mainly seen through C KLL Auger emission at $\sim 250 \mathrm{eV}$ kinetic energy while photo emitted $\mathrm{C} 1 \mathrm{~s}$ electrons with $\sim 970 \mathrm{eV}$ kinetic energy was close to the detection limit. The strongest component seen in photoemission spectra is $\mathrm{F} 1 \mathrm{~s}$ at $687.7 \mathrm{eV}$. $\mathrm{O} 1 \mathrm{~s}$ is located at $532.5 \mathrm{eV}$, which is expected from $\mathrm{SiO}_{2}$. Between the $\mathrm{F} 1 \mathrm{~s}$ and $\mathrm{O} 1 \mathrm{~s}$ Auger electrons from $\mathrm{F}$ are detected. The lower spectrum covers the region around Si2p

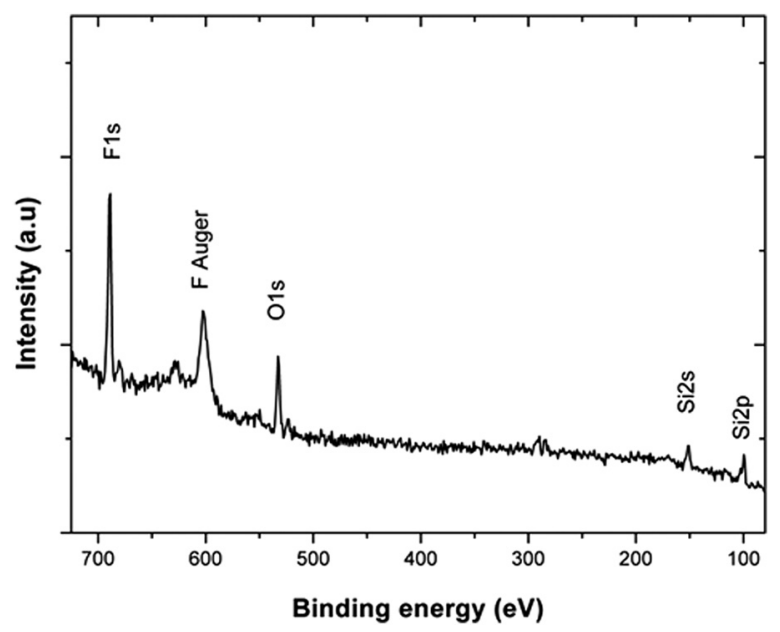

FIG. 2. XPS spectra taken from the MTS, showing strong F peaks with negligible $\mathrm{C}$ peak.
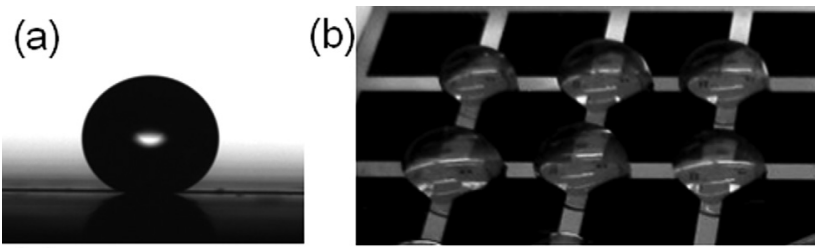

(c)

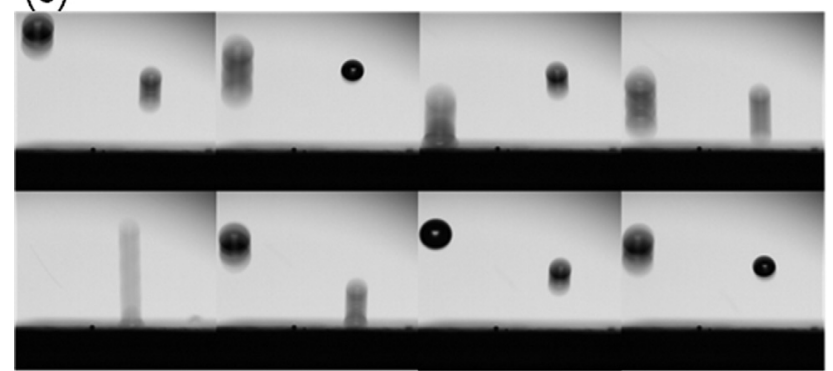

FIG. 3. Wetting behaviors of the MTS. (a) An optical image of a water drop (4 $\mu$ l) deposited on the MTS. (b) Water accumulation on non-textured silicon surface (grey) after spraying mist; the MTS shows in black. (c) Sequential images of impacting water drops $(0.5$ and $1.5 \mu \mathrm{l})$ on the MTS $(20 \mathrm{~ms} /$ frame).

and $\mathrm{Si} 2 \mathrm{~s}$. The Si2p two peaks are located at $99.3 \mathrm{eV}$ and $103.0 \mathrm{eV}$, corresponding to $\mathrm{Si}$ and $\mathrm{SiO}_{2}$. $\mathrm{Si} 2 \mathrm{~s}$ is also separated in two chemically shifted peaks.

Wetting behaviors of the MTS under static and dynamic conditions can be seen in Fig. 3. The apparent contact angle and contact angle hysteresis $168^{\circ}$ and $2^{\circ}$, respectively. The observed wetting properties are obvious because the MTS has small contact area and discontinuous three-phase contact (liquid-air-solid) line with water drop as predicted by several groups. ${ }^{13-15}$ As an alternative evidence of the low contact angle hysteresis, impacting water drops on the MTS was performed by releasing water drops at $10 \mathrm{~cm}$ height. It shows elastic bouncing-off of the impacting drops, indicating the low contact angle hysteresis (Fig. 3(c)).

Artificial superhydrophobic surfaces are commonly unstable due to instability of a composite interface caused by external stimuli such as capillary waves, nano/microdrop condensations, and surface inhomogeneity. It was suggested that hierarchical roughness is a critical element for stabilizing a composite interface. ${ }^{7-9}$ We show that the factors affected on stability of superhydrophobicity can be resolved by making hierarchical multiple air-traps, maintaining static and dynamic superhydrophobicity over one year. The MTS consists of three trapping sites for air, and those are nanocone array, micropore array, and nanogrooves. Indeed, the nanocone array like nanowire array can be regarded as an open trap for air, whereas the micropore array is a confined trap. By vertically combining two traps, possible perturbations induced by capillary waves in the open trap can be minimized since the micropore array could be functioned as a stabilizer for perturbing air. Moreover, air trapping by the nanobumps and nanogrooves resists microdrop condensation, preventing the air traps from water-filling. To clarify this, mist was sprayed on the patterned MTS, revealing that small water drops coalesce into bigger drops and eventually rolling to non-textured silicon surfaces (Fig. 3(b)).

To measure optical absorptions, we used standard hemispherical integrating system. Fig. 4 shows absorption spectra 


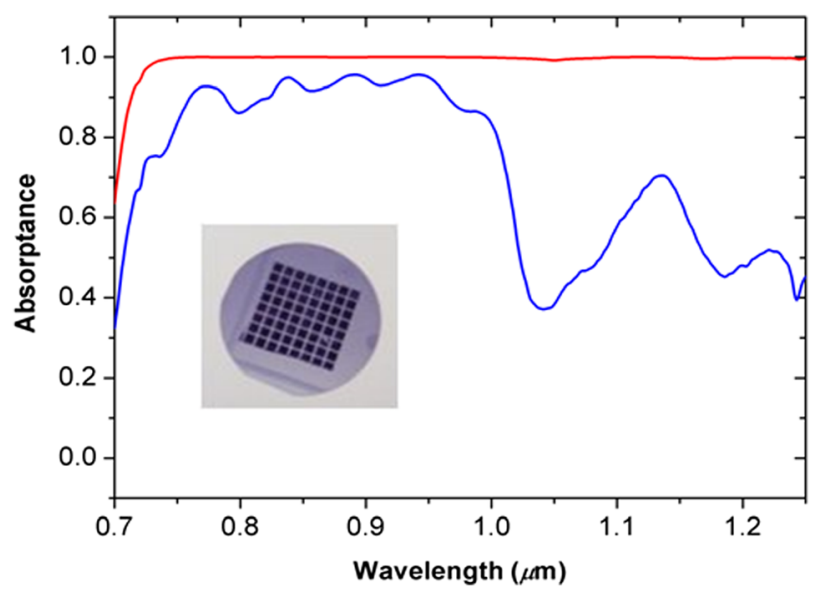

FIG. 4. (Color online) Absorption spectra of the MTS (red). Blue line shows absorption spectra after removing the nanocone array. Inset: Optical image of the MTS arrays patterned on a 4 in. silicon wafer.

of the MTS. The featureless strong absorption extends to the sub-bandgap region. Above the bandgap $(<1.1 \mu \mathrm{m})$. the strong absorption can be explained by topological effects. As such, light can be strongly confined by multiple traps. In particular, the nanocone as a graded refractive index layer reduces the reflection due to a gradual increase of the averaged reflective index. The horizontally well-ordered nanoscale bumps and grooves further enhance absorption because light is likely scattered along in-plane direction, increasing light path for absorption. However, the origin of the below bandgap absorption is unclear at present.

In the RIE process, structural defects such as fluorinevacancy complexes are formed by penetrating fluorine into silicon lattice at or near the surface structure. ${ }^{16-20}$ The defects created and the high concentration of fluorine impurity as detected by XPS can modify surface states by introducing impurity states in the bandgap. Hence, the surface of the nanocones can be considered as an absorber for photons which has energy lower than the bandgap energy.

To further investigate a role of the absorber the nanocones were removed by scrapping. Compared to the pristine MTS, it shows distinctive optical properties. The absorption decreases over a wide range of wavelengths. The reduction becomes more pronounced below the bandgap, which is caused by the reduction of trapping sites and the absorbing layer. Interestingly, oscillation of the absorption occurs, which is ascribed to optical impedance mismatching at the interface between the nanocones and the underneath micropores. It suggests that the nanocone array can be functioned as a good optical impedance matching system for higher absorption. In conjunction with the optimum optical impedance matching system produced by the MTS, the defectinduced absorbing layer is revealed as a novel platform for enabling the strong absorption below the bandgap.

In summary, we have demonstrated that a well-defined multilayer decorated by nanostructures as exhibited in a wing of male Papilio ulysse could be a direct means of effective entrapment of air and light, resulted in stable superhydrophobic under static and dynamic conditions, and strong wideband optical absorption. Our results highlight that the unique characteristics were simultaneously obtained from the structure prepared by using simple microelectromechanical technique, which can readily build integrated systems for electro-optical devices. The pertaining self-cleaning property provides further protective layer on the devices. Owing to the well-defined structure, it can be used as a model structure for studying wetting and optical properties in surfaces of biological relevance.

This work was supported by the SSF (Swedish Foundation for Strategic Research), No. RMA08-0090.

${ }^{1}$ P. Vukusic, J. R. Sambles, and C. R. Lawrence, Proc. R. Soc. London, Ser. B 271, S237 (2004).

${ }^{2}$ S. Berthier, Iridescences: The physical Colors of Insects (Springer, New York, 2007).

${ }^{3}$ G. J. Parker, J. Mater. Sci.: Mater. Electron. 21, 965 (2010).

${ }^{4}$ A. B. D. Cassie and S. Baxter, Trans. Faraday Soc. 40, 546 (1944).

${ }^{5}$ A. Checco, P. Guenoun, and J. Daillant, Phys. Rev. Lett. 91, 166101 (2003).

${ }^{6}$ Y. P. Cheng, D. E. Rodak, A. Angelopoulos, and T. Gacek, Appl. Phys. Lett. 8, 194112 (2005).

${ }^{7}$ M. Nosonovsky and B. Bhushan, Microsyst. Technol. 12, 231 (2006).

${ }^{8}$ M. Nosonovsky, Langmuir 23, 3157 (2007).

${ }^{9}$ B. Bhushan and M. Nosonovsky, Philos. Trans. R London, Ser. A 368, 4713 (2010).

${ }^{10}$ F. Laermer and A. Schilp, U.S. patent 5501893, March 26 (1996).

${ }^{11}$ A. M. Hynes, H. Ashraf, J. K. Bhardwaj, J. Hopkins, I. Johnston, and J. N. Shepherd, Sens. Actuators 74, 13 (1999).

${ }^{12}$ K. T. Tamamori, T. Itsuji, S. Wang, R. Kurosaka, and H. Ono, Jpn. J. Appl. Phys. 46, L654 (2007).

${ }^{13}$ E. L. Decker and S. Garoff, Langmuir 13, 6321 (1997).

${ }^{14}$ N. Anantharaju, M. V. Panchagnula, S. Vedantam, S. Neti, and S. TaticLucic, Langmuir 23, 11673 (2007).

${ }^{15}$ L. Gao and T. J. McCarthy, Langmuir 23, 3762 (2007).

${ }^{16}$ X. D. Pi, C. P. Burrows, and P. G. Coleman, Phys. Rev. Lett. 90, 155901 (2003).

${ }^{17}$ M. Diebel and S. T. Dunham, Phys. Rev. Lett. 93, 245901 (2004).

${ }^{18}$ S. A. Harrison, T. F. Edgar, and G. S. Hwang, Phys. Rev. B 74, 121201 (2006).

${ }^{19}$ G. M. Lopez and V. Fiorentini, Appl. Phys. Lett. 89, 092113 (2006).

${ }^{20}$ H. Winters and D. B. Graves, J. Vac. Sci. Technol. A 25, 96 (2007). 